\title{
Dried plasma spots in the diagnosis of tuberculosis: IP-10 release assay on filter paper
}

\author{
Martine G. Aabye1, Irene Latorre², Jessica Diaz², Jose Maldonado3, \\ Irene Mialdea ${ }^{4}$, Jesper Eugen-Olsen ${ }^{1}$, Pernille Ravn ${ }^{1,5}$, Jose Dominguez ${ }^{2}$ and \\ Morten Ruhwald ${ }^{1}$
}

\begin{abstract}
Affiliations: ${ }^{1}$ Clinical Research Centre, Copenhagen University Hospital, Hvidovre, Copenhagen, and ${ }^{5}$ Dept of Infectious Diseases, University Hospital, Hillerød, Denmark. ${ }^{2}$ Servei de Microbiologia, Hospital Universitari Germans Trias i Pujol, Institut d'Investigació en Ciències de la Salut Germans Trias i Pujol, Universitat Autònoma de Barcelona, CIBER Enfermedades Respiratorias, Instituto de Salud Carlos III, Badalona, ${ }^{3}$ Serveis Clinics, Barcelona, and ${ }^{4}$ Hospital Clínico Universitario de Valencia, Valencia, Spain.
\end{abstract}

Correspondence: M. Ruhwald, Clinical Research Centre, Copenhagen University, Hvidovre Hospital, Kettegaards Alle 30, DK-2650 Copenhagen, Denmark. E-mail: mruhwaldagmail.com

ABSTRACT Interferon (IFN)- $\gamma$ release assays (IGRAs) are probably the most accurate tests for the detection of latent Mycobacterium tuberculosis infection, but IGRAs are labour intensive and the transport of samples over longer distances is difficult. IFN- $\gamma$-induced protein (IP)-10 is expressed at 100 -fold higher levels than IFN- $\gamma$, and IP-10 release assays have comparable performance to IGRAs. The aim of this study was to explore the diagnostic potential of a novel IP-10 release assay based on dried plasma spots (DPS).

The presence of IP-10 and IFN- $\gamma$ was determined in plasma and in DPS by ELISA. Diagnostic algorithms for plasma and DPS tests for IP-10 were developed on a training cohort comprising 60 tuberculosis (TB) patients and 59 healthy controls. Diagnostic accuracy was assessed in a validation cohort comprising $78 \mathrm{~TB}$ patients and 98 healthy controls. Plasma was measured in Spain and DPS samples were sent to Denmark using the conventional postal service for analysis.

IP-10 was readily detectable in both plasma and DPS, and correlation was excellent $\left(\mathrm{r}^{2}=0.95\right)$. QuantiFERON-TB Gold In-Tube (QFT-TB) and IP-10 in DPS and plasma rendered comparable sensitivity (78\%, $82 \%$ and $84 \%$, respectively), specificity $(100 \%, 97 \%$ and $97 \%$, respectively) and indeterminate rates $(\mathrm{p}>0.55)$.

The DPS-based IP-10 test has comparable diagnostic accuracy to the QFT-TB and samples can be sent via conventional mail over long distances for analysis without affecting the results.

@ERSpublications

The dried plasma spot-based IP-10 test has comparable diagnostic accuracy to IFN- $\gamma$ release assays http://ow.ly/kNSMI

Received: Aug 172012 | Accepted after revision: Sept 272012 | First published online: Jan 242013

Support statement: This work was supported by the Danish National Advanced Technology Foundation (003-2011-5), the Capital Region of Copenhagen, the Danish Lung Association, the Danish Ministry of Science, Innovation and Higher Education, the Clinical Research Centre at Hvidovre Hospital and the Lundbeck Foundation (R32-A1081) (Denmark). Jose Domínguez is funded by the Miguel Servet programme of the Instituto de Salud Carlos III (Badalona, Spain).

Conflict of interest: Disclosures can be found alongside the online version of this article at www.erj.ersjournals.com

Copyright CERS 2013. ERJ Open articles are open access and distributed under the terms of the Creative Commons Attribution Non-Commercial Licence 3.0.

This article was modified in April 2016 to correct errors in the licence information. 


\section{Introduction}

Interferon (IFN) $-\gamma$ release assays (IGRAs) are probably the most accurate diagnostic tests for latent tuberculosis infection (LTBI) [1]. There are two IGRAs on the market, the T-SPOT.TB (Oxford Immunotec, Abingdon, UK) and the QuantiFERON-TB Gold In-Tube (QFT-TB; Cellestis, Chadstone, Australia). Both tests utilise the ability of sensitised T-cells to release IFN- $\gamma$ when stimulated with peptides from Mycobacterium tuberculosis-specific antigens in vitro. While the first method measures the frequency of reactive lymphocytes in the peripheral blood mononuclear cell fraction, the latter measures the concentration of released IFN- $\gamma$ in plasma of whole blood $[2,3]$. The IGRAs address two of the major limitations of the tuberculin skin test: low specificity in bacille Calmette-Guerin (BCG)-vaccinated subjects and logistical challenges, with tests having to be read 2-3 days after application [4].

There are major obstacles with implementation of the IGRA, especially in low-resource settings [2]: the TSPOT.TB requires access to skilled technicians to separate cells and to set up the assay for incubation. The window of time between blood sampling and laboratory analysis is short and requires that the blood be transported to the laboratory at ambient temperature. For the QFT-TB, incubation is done directly in the blood collection tubes, and following a centrifugation step, samples can be transported refrigerated or frozen. Transport of these samples requires a cold chain and safety precautions must be considered, as the plasma is potentially infectious. Simpler, safer and more flexible solutions are needed in both high- and lowresource regions.

We have challenged these obstacles using the biomarker IFN- $\gamma$-inducible protein (IP)-10. IP-10 is a chemokine expressed in concert with IFN- $\gamma$ in the IGRA [5]. The diagnostic accuracy of the IP-10 release assay has been assessed in 26 clinical studies and it is established that the IP-10 release assay performs at a par with an IGRA based on IFN- $\gamma$ [5-7]. In contrast to the T-cell derived IFN- $\gamma$, IP-10 is mainly secreted from the antigen presenting cells. IP-10 expression is stimulated by multiple signals downstream of the Tcell signals, mainly IFN- $\gamma$ and tumour necrosis factor- $\alpha$, but also interleukin (IL)-2, type II IFNs, IL-27, IL17/IL-23 and IL-1 $\beta[6,8-10]$, supporting pathogen specific adaptive immune responses by a positive feedback loop [11]. In vitro, IP-10 is released in 100-fold higher levels than IFN- $\gamma$ [12], which allows for alternative methods of storing and transportation of samples.

Blood or plasma blotted onto filter paper has been shown to be a safe, robust and convenient method for the collection, transport and storage of small volumes of samples for analysis of biomarkers, for example [13]. In preparation for this study we developed and validated an ELISA assay for IP-10 detection both for plasma samples and for dried blood and plasma spots (DBS and DPS, respectively) from unstimulated, QFT-TB- and cytomegalovirus-stimulated whole blood [12].

The aim of this study was to investigate whether an IP-10 release assay using whole blood stimulated with antigens specific for M. tuberculosis stored as DPS could be used to diagnose M. tuberculosis infection. We applied the method to two sets of patients with confirmed tuberculosis (TB) and healthy controls. We defined cut-off points and established a diagnostic algorithm based on the first set of patients and controls; subsequently we evaluated the test performance in the other set of cases and controls.

\section{Methods}

Study participants

For establishing the cut-off and diagnostic algorithm we included a training set comprising 60 microbiologically confirmed patients with active TB at the Copenhagen University Hospital (Hvidovre, Denmark) and 59 healthy unexposed Danish controls (table 1). The controls were included as part of a larger contact investigation at a high-school in Copenhagen [14]. This investigation demonstrated very limited exposure to nonclassmates, and we included the controls among nonclassmates without travel, family or contact exposure. Evaluation of test performance was conducted in the validation set comprising 78 microbiologically confirmed TB patients from Barcelona, Spain and 107 healthy secondary school students from Valencia, Spain. The controls were included as part of the routine screening of Valencia secondary school students and the majority of the students $(61 \%)$ were recent immigrants or children of recent immigrants. Students were consecutively included and those who reported known TB exposure, previous TB treatment or lymphadenitis of unknown aetiology were excluded ( $n=9$ out of 107 screened). The patients used for validation thus comprised 78 patients and 98 healthy controls.

\section{Blood stimulation and QFT-TB testing}

All participants had a QFT-TB test performed. IFN- $\gamma$ levels were determined according to the QFT-TB package insert and excess plasma was stored at $-80^{\circ} \mathrm{C}$ for IP-10 measurements and spotting on filter paper. 


\begin{tabular}{|c|c|c|c|c|}
\hline & \multicolumn{2}{|c|}{ Training set } & \multicolumn{2}{|c|}{ Validation set } \\
\hline & TB & Control & TB & Control \\
\hline Subjects $n$ & 60 & 59 & 78 & 98 \\
\hline Age years & $40(31-52)$ & $17(15-18)$ & $35(26-45)$ & $14(13-15)$ \\
\hline Males & $40(67)$ & $55(50)$ & $56(72)$ & $49(50)$ \\
\hline \multicolumn{5}{|l|}{ Place of birth } \\
\hline South America & $2(3)$ & $0(0)$ & 15 (19) & $50(51)$ \\
\hline Eastern Europe & $9(15)$ & $0(0)$ & $3(4)$ & $5(5)$ \\
\hline Asia & $6(10)$ & $0(0)$ & $11(14)$ & $4(4)$ \\
\hline Unknown & $1(2)$ & $0(0)$ & $5(6)$ & $0(0)$ \\
\hline \multicolumn{5}{|l|}{ BCG status } \\
\hline Yes & $0(0)$ & $0(0)$ & $10(13)$ & $59(60)$ \\
\hline Extrapulmonary TB & $26(29)$ & $0(0)$ & $6(8)$ & $0(0)$ \\
\hline \multicolumn{5}{|l|}{ Comorbidity } \\
\hline None & $53(87)$ & $59(100)$ & $60(77)$ & $98(100)$ \\
\hline Diabetes mellitus & $0(0)$ & $0(0)$ & $5(6)$ & $0(0)$ \\
\hline $\mathrm{HCV}$ & $0(0)$ & $0(0)$ & $5(6)$ & $0(0)$ \\
\hline HIV & 7 (13) & $0(0)$ & $3(4)$ & $0(0)$ \\
\hline $\mathrm{HIV}+\mathrm{HCV}$ & $0(0)$ & $0(0)$ & $2(3)$ & $0(0)$ \\
\hline $\mathrm{HIV}+\mathrm{HBV}$ & $0(0)$ & $0(0)$ & $1(1)$ & $0(0)$ \\
\hline Renal transplant + steroids & $0(0)$ & $0(0)$ & $1(1)$ & $0(0)$ \\
\hline \multicolumn{5}{|l|}{ Tests } \\
\hline TST positive/tested & $0 / 0(0)$ & $0 / 53(0)$ & $16 / 28(57)$ & 18/96 (19) \\
\hline TST $\mathrm{mm}$ & $0.0(0-0)$ & $0.0(0-0)$ & $15(10-18)$ & $9(6-12)$ \\
\hline
\end{tabular}

Data are presented as median (interquartile range) or $\mathrm{n}$ (\%), unless otherwise stated. BCG: bacille Calmette-Guérin; TB: tuberculosis; HCV: hepatitis C virus; HBV: hepatitis B virus; TST: tuberculin skin test; NAAT: nucleic acid amplification test; NA: not available.

Filter paper preparation and storage

QFT-TB stimulated plasma supernatant $(25 \mu \mathrm{L})$ was spotted in duplicates on filter paper (903 Protein Saver ${ }^{\mathrm{TM}}$ cards; Whatman plc, Maidstone, UK), dried at ambient temperature for $4 \mathrm{~h}$ and stored in plastic bags with desiccant. Training set samples were stored on filter paper at $5^{\circ} \mathrm{C}$ for 10 days before analysis; validation set samples were stored for $2-4$ days at $5^{\circ} \mathrm{C}$ before transport via normal 2-day postal service at ambient temperature from Spain to Denmark for analysis. Analysis was performed 2 days after arrival.

\section{IP-10 ELISA (plasma and DPS)}

We used an in-house ELISA for IP-10 detection [12]. In brief, we developed rat and murine hybridoma cell lines producing monoclonal antibodies (mAbs) specific for IP-10. mAbs were combined in a sandwich ELISA which was optimised for the range of IP-10 responses in QFT-TB samples in both plasma and DPS.

For the assay, plasma samples were diluted $\times 33$ in assay buffer with horseradish peroxidise (HRP)conjugated detection $\mathrm{mAb}$ (corresponding to $3 \mu \mathrm{L}$ plasma per $100 \mu \mathrm{L}$ ELISA well). For DPSs, two circular discs $5.5 \mathrm{~mm}$ in diameter were punched from the centre of the DPS using a standard office paper-puncher (Impega, Telford, UK), DPS discs were placed in the ELISA plate well and $100 \mu \mathrm{L}$ assay buffer was added. Diluted plasma samples and DPS discs were analysed using the same protocol, i.e. incubation for $2 \mathrm{~h}$ at room temperature and washed three times. HRP-substrate (TMB One; Trichem, Roskilde, Denmark) was added, plates were revealed for $30 \mathrm{~min}$, the enzyme reaction was stopped and absorbance was read at $450 \mathrm{~nm}$, subtracted readings at $630 \mathrm{~nm}$. Concentrations were calculated using a standard curve generated for each ELISA plate in duplicates and fitted with linear regression of the expected concentrations against the mean optical density. IP-10 in plasma was measured in duplicate and the mean was used for further 
analysis. IP-10 in DPS samples was determined by single measurements. The linear range of the ELISA is 2.5-600 pg. $\mathrm{mL}^{-1}$; a full description of the performance characteristics of the ELISA is available in [12]. Plasma levels of IP-10 are presented after multiplication by 33 to compensate for dilution, DPS levels are presented as pg detected per two 5.5- $\mathrm{mm}$ discs (pg per 2 discs). All samples were analysed blinded from the clinical information.

\section{Logistics}

All ELISA reagents were prepared in Copenhagen, Denmark. Plasma samples were measured in Barcelona, Spain (validation set) and Copenhagen (training set). DPS were exclusively measured in Copenhagen.

\section{Ethics}

The study was approved by the Ethical Committee of Copenhagen (KF-01-278477), and by the ethical committee of the Hospital Universitari Germans Trias i Pujol, Barcelona.

\section{Statistics}

Biomarker levels were compared using nonparametric paired and nonpaired methods (Wilcoxon signed rank test, Mann-Whitney U-test and Spearman). Cut-offs were set using receiver operating characteristic (ROC) curve analysis (at high sensitivity with minimal loss of specificity) and test concordance was compared using McNemar's test and $\kappa$-statistics. Sensitivity and specificity were calculated after omission of indeterminate responders. A p-value $<0.05$ was considered significant. Analyses were performed using SAS 9.2 (SAS Institute Inc., Cary, NC, USA) and GraphPad Prism 5.0 (GraphPad Software Inc., La Jolla, CA, USA).

\section{Results}

Comparison of IP-10 in plasma and DPS and defining diagnostic test cut-offs using training set samples

We compared IP-10 DPS and plasma samples in the training cohort comprising 60 patients with active TB and 59 healthy controls. Compared to controls, the TB patients were older, and $62 \%$ were born in countries outside Western Europe (clinical data available in table 1). The nil levels and antigen-induced IP-10 and IFN- $\gamma$ responses were significantly higher in TB patients compared to controls, and mitogen responses were lower (table 2). There was an excellent correlation between plasma and DPS IP-10 $\left(\mathrm{r}^{2}=0.95\right)$ (fig. 1), while the correlation between IFN- $\gamma$ and IP-10 was only moderate $\left(r^{2}<0.48\right.$; data not shown).

We compared the diagnostic potential of the three assays using ROC curve analysis and found comparable areas under the curve (AUCs): 0.95, 0.94 and 0.89 for IP-10 plasma, IP-10 DPS and QFT-TB, respectively (fig. 2) with no significant difference between the assays. ROC analysis suggested the following cut-off values for an assay with high specificity: IP-10 plasma $2.3 \mathrm{ng} \cdot \mathrm{mL}^{-1}$ (sensitivity $87 \%$, specificity $100 \%$ ) and IP-10 DPS 105 pg per 2 discs (sensitivity 87\%, specificity 100\%). For comparison, the optimal cut-off for QFT-TB on these data was $0.21 \mathrm{IU} \cdot \mathrm{mL}^{-1}$ (sensitivity $83 \%$, specificity $100 \%$ ). Vis à vis the option in the

TABLE 2 Interferon (IFN)- $\gamma$-inducible protein (IP)-10 in plasma and dried plasma spots (DPS) and IFN- $\gamma$ in plasma in the training and validation sets

Training set

Controls TB patients

\section{Validation set}

\begin{tabular}{lll}
\hline Controls & TB patients & p-value
\end{tabular}

\begin{tabular}{|c|c|c|c|c|c|c|}
\hline \multicolumn{7}{|c|}{ IFN- $\gamma$ plasma IU $\cdot \mathrm{mL}^{-1}$} \\
\hline Nil & $0.05(0.04-0.08)$ & $0.15(0.07-0.36)$ & $<0.0001$ & $0.00(0.00-0.06)$ & $0.17(0.05-0.29)$ & $<0.0001$ \\
\hline Antigen-nil & $0.01(0.00-0.02)$ & $2.68(0.42-6.42)$ & $<0.0001$ & $0.00(0.00-0.05)$ & $1.74(0.30-4.86)$ & $<0.0001$ \\
\hline Mitogen-nil & $8.61(7.98-9.94)$ & $6.39(0.76-9.9)$ & $<0.0001$ & $10.00(9.89-10.00)$ & $3.71(1.13-9.71)$ & $<0.0001$ \\
\hline \multicolumn{7}{|c|}{ IP-10 plasma $\mathrm{ng} \cdot \mathrm{mL}^{-1}$} \\
\hline Nil & $0.0(0.0-0.2)$ & $0.6(0.3-1.4)$ & $<0.0001$ & $0.2(0.0-0.3)$ & $0.8(0.4-1.5)$ & $<0.0001$ \\
\hline Antigen-nil & $0.1(0.0-0.3)$ & $8.1(3.6-15.8)$ & $<0.0001$ & $0.2(0.0-0.4)$ & $8.3(2.8-17.3)$ & $<0.0001$ \\
\hline Mitogen-nil & $9.3(6.7-14.5)$ & $6.3(2.7-11.6)$ & 0.0024 & $13.8(9.5-17.3)$ & $7.5(3.6-12.3)$ & $<0.0001$ \\
\hline \multicolumn{7}{|c|}{ IP-10 DPS pg per 2 discs } \\
\hline Nil & $6(2-17)$ & $38(20-69)$ & $<0.0001$ & $8(5-19)$ & $35(16-77)$ & $<0.0001$ \\
\hline Antigen-nil & $5(0.0-11)$ & $313(171-567)$ & $<0.0001$ & $5(0.0-17)$ & 355 (137-512) & $<0.0001$ \\
\hline Mitogen-nil & 401 (287-729) & $287(113-478)$ & 0.0112 & 467 (388-589) & 331 (231-587) & 0.0004 \\
\hline
\end{tabular}

Data are presented as median (interquartile range), unless otherwise stated. TB: tuberculosis. 
FIGURE 1 Comparison of interferon- $\gamma$ inducible protein (IP)-10 detected in dried plasma spots (DPS) and plasma. DPS and plasma samples from nil, antigen and mitogen samples from the training cohort comprising 60 patients with active TB and 59 healthy controls were compared. There was an excellent correlation between plasma and DPS IP-10 (Spearman $\left.\mathrm{r}^{2}=0.95\right)$.

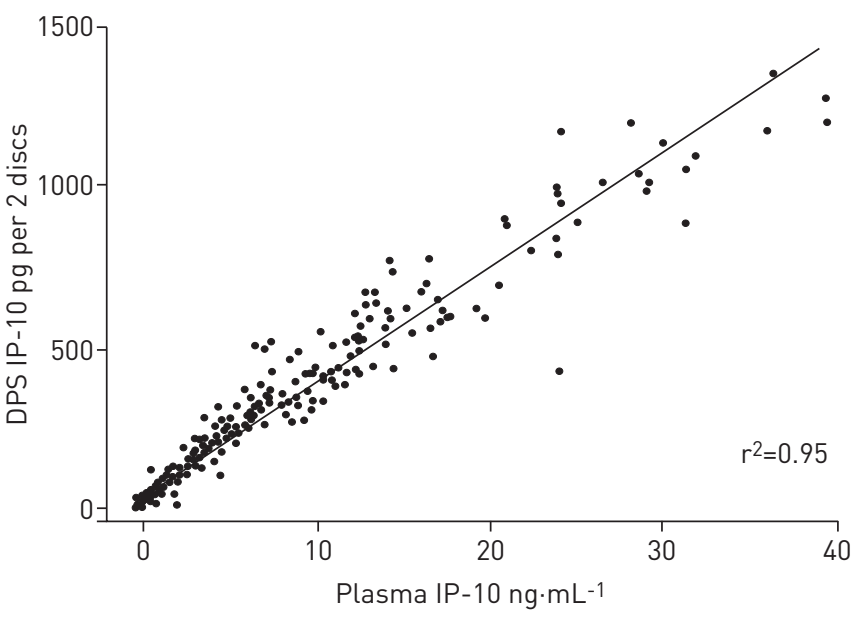

QFT-TB test algorithm, we defined a cut-off for an indeterminate test. We chose the cut-off values for an indeterminate test at the highest mitogen response value that did not result in indeterminate responders among the controls: $1.5 \mathrm{ng} \cdot \mathrm{mL}^{-1}$ for IP-10 in plasma and $71 \mathrm{pg}$ per 2 discs for IP-10 in DPS. For analysis of IFN $-\gamma$ results, the QFT-TB algorithm was used as recommended by the manufacturer.

\section{Validation of the diagnostic performance of the DPS-based test using clinical samples}

We validated the diagnostic potential of the DPS and plasma IP-10 test algorithms in a new cohort of 78 patients with culture-confirmed active TB and 98 healthy controls. Patients were older than the controls $(\mathrm{p}<0.0001)$, and more of them were male $(\mathrm{p}<0.0001) .17$ patients had a comorbid condition (table 1$)$. All but two controls were TST tested, of which 19\% were TST-positive (cut-off $10 \mathrm{~mm}$ for nonvaccinated and $15 \mathrm{~mm}$ for BCG-vaccinated subjects), and 61\% were BCG vaccinated (table 1).

The correlation between plasma levels of IP-10 and filter paper levels of IP-10 was excellent $\left(r^{2}=0.93\right.$; $\mathrm{p}<0.0001$ ) (data not shown). Both methods gave ROC curves with similar AUCs of 0.89-0.91 (data not shown) for IP-10 as well as plasma IFN- $\gamma$.

\section{Test accuracy and concordance}

We applied the IP-10 cut-off values for positive and indeterminate test results to the validation set and found that the three tests rendered comparable sensitivity (78-84\%), specificity (97-100\%) and indeterminate rate $(<1 \%$ in controls and $5 \%, 10 \%$ and $8 \%$ for QFT-TB, IP-10 DPS and IP-10 plasma, respectively, in TB patients; $\mathrm{p}>0.55$ ) (table 3 ). For comparison, we also applied the cohort-specific optimal cut-off for QFT-TB at $0.21 \mathrm{IU} \cdot \mathrm{mL}^{-1}$; the sensitivity was $82 \%$ (61 out of 74 subjects) and the specificity was $97 \%$ ( 95 out of 98 subjects); none of the controls and four (5\%) of the TB patients had indeterminate results. Agreement between DPS and plasma IP-10 tests was very high $(\kappa=0.91 ; 95 \%$ CI $0.88-0.99)$, and higher than the agreement between the QFT-TB test and IP-10 in plasma $(\kappa=0.83 ; 95 \%$ CI $0.75-91)$.

FIGURE 2 Receiver operating characteristic curve analysis. Antigen-specific release of interferon (IFN) $-\gamma$ and IFN$\gamma$-induced protein (IP)-10 was compared in training set samples. IP-10 was detected in dried plasma spot (DPS) and plasma samples, IFN- $\gamma$ in plasma using the QuantiFERON-TB Gold InTube (QFT-TB) ELISA. Areas under the curve were comparable, at 0.95 , 0.94 and 0.89 for IP-10 plasma, IP-10 DPS and QFT-TB, respectively.

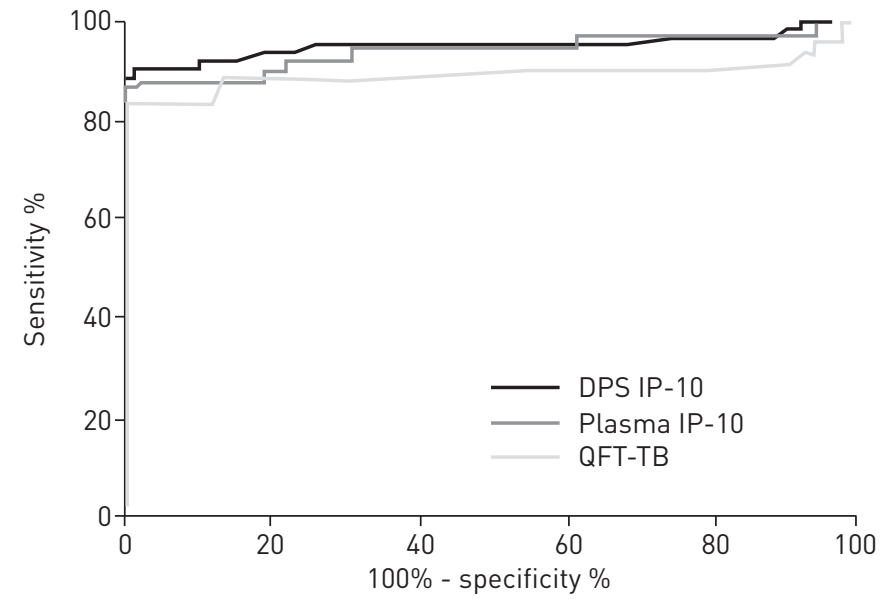


Combining plasma IP-10 results with QFT-TB test results (i.e. a positive result in either or both tests is considered positive; indeterminate if one test is indeterminate and the other is not positive) increased the sensitivity significantly to $89 \%(\mathrm{p}=0.032)$ without a compromise in specificity.

\section{Discussion}

We investigated the potential of combining the user-friendly filter paper method with an IP-10 release assay with the purpose of diagnosing infection with M. tuberculosis. We demonstrated that it is possible to dry plasma from QFT-TB tubes on filter paper, transport samples across Europe at ambient temperature in a letter, and extract the diagnostic information without either loss in signal strength or diagnostic accuracy.

IP-10 is an emerging alternative biomarker to IFN- $\gamma$ for cell-mediated immune response assays such as the IGRA $[5,6,15]$. The findings of this study support a series of recent papers which have demonstrated that IP-10 release assays have comparable diagnostic accuracy with the QFT-TB in TB patients versus controls [6, $16,17]$. Other studies have suggested that IP-10 has superior diagnostic performance in HIV-infected patients, independent of low CD4 count [18-22]; in patients with autoimmune diseases [23]; in young children [24-27]; and in patients suspected of having active TB who had been diagnosed with other bacterial or viral infections [16]. A consistent finding in all studies is a slight discordance between IP-10 and IFN $-\gamma$ responders, which opens the possibility of combining the markers for increased sensitivity, seemingly without affecting specificity $[16,17]$.

The concept of spotting blood onto filter paper for diagnostic purposes was introduced by Guthrie and Susi in 1963 as a tool to screen for metabolic diseases in large populations of neonates [13]. The dried blood or plasma matrix stabilises many analytes, and allows for determination of both phenotype (e.g. biomarkers) and genotype (e.g. polymorphisms) from a single specimen $[13,28]$. Transporting samples dried on filter paper is considered safe [13] and the Whatman 903 filter paper is approved by the US Food and Drug Administration (class II medical device). Neonatal screening using DBS is a standard of care worldwide [29] and the filter paper technology is also used for hepatitis C and HIV viral load monitoring and HIV screening in many countries, including South Africa [28].

We have developed the filter paper based method for IP-10 detection in stimulated and unstimulated whole blood using a simple ELISA based assay. For optimal detection of IP-10 in antigen-stimulated samples we diluted plasma extensively $\times 33$ times $(3 \mu \mathrm{L}$ plasma per ELISA plate well). The signal intensity generated from this low volume corresponds well to IP-10 signals generated from two DPS or DBS discs, and from the $50-\mu \mathrm{L}$ plasma sample used for IFN- $\gamma$ detection in the QFT-TB ELISA. We recently reviewed 22 clinical studies on IP-10 release assays for the diagnosis of infection with M. tuberculosis. We found that IP-10 had been detected by five very different IP-10 assays and at sample dilutions from $\times 2$ to $\times 20$ [6]. This heterogeneity limits comparison between assays and calls for consensus in the field. The easy sample transport enabled by the filter paper method is ideal for international collaborations and larger multicentre studies, and should lead to better quality and comparable studies on IP-10 release assays.

SKOGSTRAND and colleagues $[30,31]$ pioneered the measurement of cytokines and chemokines in dried blood spots using a Luminex based multiplex approach, and have validated clinical use in a series of studies of inflammatory conditions from neonates using heel blood samples. Recently, a small proof-of-concept study assessed the multiplex assay using DBS samples from QFT-TB stimulated blood [32]. This study demonstrated a significant difference in IFN- $\gamma$ responses between QFT-TB positive patients and QFT-TB negative patients, but the majority of samples, also from $\mathrm{TB}$ patients, fell below the lower limit of

TABLE 3 Test results

IP-10 plasma

IP-10 DPS

QFT-TB

IP-10 plasma + QFT-TB

\begin{tabular}{|c|c|c|c|c|}
\hline \multicolumn{5}{|l|}{ TB patients } \\
\hline Positive & $61(78)$ & $58(74)$ & $58(74)$ & $64(82)$ \\
\hline Negative & $11(14)$ & $12(15)$ & $16(21)$ & $8(11)$ \\
\hline Indeterminate & $6(8)$ & $8(10)$ & $4(5)$ & $6(8)$ \\
\hline \multicolumn{5}{|l|}{ Controls } \\
\hline Positive & $2(2)$ & $2(2)$ & $0(0)$ & $2(2)$ \\
\hline Negative & 95 (97) & 95 (97) & 98 (100) & 95 (97) \\
\hline Indeterminate & $1(1)$ & $1(1)$ & $0(0)$ & 1 (1) \\
\hline
\end{tabular}

Data are presented as n (\%). IP: interferon- $\gamma$-induced protein; DPS: dried plasma spot; QFT-TB: QuantiFERON-TB Gold In-Tube test; TB: tuberculosis. 
quantification of the assay [33]. We have attempted to extract IFN- $\gamma$ signals from DPS samples using the QFT-TB ELISA, but also failed to detect reliable responses [12]. The likely explanation for these results is that the low volume of plasma available in the filter paper samples contains too little IFN- $\gamma$ for reliable determination. IP10 is expressed in 100-fold higher levels and is therefore better suited for this method [12, 34].

\section{Limitations}

Our long-term aim is to develop a simple test for LTBI, but as there is no true gold standard for LTBI we used patients with active TB as a proxy for M. tuberculosis infection. This approach provides a solid gold standard for infection, but patients with active TB disease may have compromised IFN- $\gamma$ and IP-10 responses to mitogens, and probably also to antigens which could lead to inaccurate cut-off values for positive test results if used for LTBI or in patients with immunosuppression, e.g. HIV infected patients $[16,35,36]$. Nevertheless, as described in detail by ZHOU et al. [37], evaluation of new diagnostic tests (or diagnostic algorithms) should be performed in several steps; first on selected populations in order to ensure assessment of sensitivity and specificity under (for the test) optimal conditions before the test can be applied in a clinical setting, e.g. a prospective study evaluating the predictive values of the test. The "gold standard problem" in assessing new tests for LTBI is inherent to the field [38], and all tests for LTBI, including the QFT-TB test, have been assessed using a similar case-control design [39].

Our sample size in the training set only comprised 119 patients and controls. This relatively small cohort will result in an imprecise assessment of cut-off values. But, as we found comparable diagnostic accuracy between the training and the validation set, despite controls from very different settings, we suspect this risk to be limited.

Furthermore, the age difference between patients and controls both within and across the training and validation cohorts was significant. These differences might have influenced the findings, but we believe the impact to be minor because our youngest controls were teenagers (and not $<5$-year-olds, which is the only age group with documented impaired performance of IGRA), and because IP-10 appears to be less influenced by young age compared to IFN- $\gamma[24,27]$. Nevertheless, further studies are needed and these should be designed to allow for adjustments of the cut-off value for a positive test result. In contrast to other studies in similar groups of patients and controls, we observed a higher number of patients with indeterminate IP-10 responses compared to QFT-TB [16, 17]; this most probably reflects a relatively lower threshold for an indeterminate test result in our study. Previous studies on IP-10 have used arbitrary values to mimic the rates seen for QFT-TB [6] and the method used to set the QFT indeterminate cut-off is, to our knowledge, not published $[39,40]$. In this study we set the cut-off for indeterminate values just below the lowest IP-10 mitogen response observed in the cohort of healthy controls; this to optimise sensitivity (by deeming patients with false negative responses to be indeterminate) without compromising costeffectiveness (deeming true negative controls as indeterminate). Higher cut-off values rendered indeterminate rates at par with the QFT-TB (data not shown). Interestingly, the lower threshold for an indeterminate IP-10 test result did not affect the improvements in accuracy seen when combining IP-10 and QFT-TB [16-18, 41, 42]. Further studies are needed to evaluate and potentially adjust the cut-off, and to assess the risk of developing active TB given a false negative/indeterminate test result.

The findings of this study call for confirmation in other populations, e.g. latent versus active TB; and assessments of the performance of the method in HIV infected subjects and children, as well as prospective evaluations of the positive and negative predictive value of IGRAs based on IFN- $\gamma$ and IP-10, are urgently needed.

\section{Perspectives}

The filter paper method for plasma sample storage and transport enables simplification and centralisation of the IGRA diagnostic concept. IP-10 is detectable in DBS samples in comparable levels to DPS samples, whereby stimulated samples can be prepared without the centrifugation step [12]. With a simpler test format the workload and requirements of local laboratories will make IP-10 release assays easier and cheaper to set up, e.g. for low-volume users, and peripheral or resource restricted settings. Importantly, the easy sample transport allows for better quality control of the analysis, e.g. the possibility for employing a reference laboratory function. Fully automated high-throughput systems for filter paper punching and sample analyses are standard for neonatal screening, and can be adapted for IP-10 measurement [29]. An automated analysis significantly reduces the risks of human error and assay variability linked to performing manual ELISA and ELISPOT assays, and increases analytical precision and diagnostic accuracy.

\section{Conclusion}

This study describes and validates a method for simplifying sample handling in an IP-10 release assay for the diagnosis of M. tuberculosis infection. We show that IP-10 is stable in plasma dried on filter paper, and can be sent with normal mail over long distances for analysis, apparently without affecting the results. Also, the 
study adds further to the evidence that IP-10 release assays are a valid alternative to IGRAs, and that such IP-10 release assays have operational advantages using the filter paper approach.

\section{Acknowledgements}

The authors would like to thank Ove Andersen (Clinical Research Centre, Copenhagen University Hospital, Hvidovre, Copenhagen, Denmark) for support and for providing the framework; and TB-NET (Borstel, Germany) for promoting a trans-European research collaboration, without which this work would not have happened.

\section{References}

1 Diel R, Goletti D, Ferrara G, et al. Interferon- $\gamma$ release assays for the diagnosis of latent Mycobacterium tuberculosis infection: a systematic review and meta-analysis. Eur Respir J 2011; 37: 88-99.

2 Lalvani A, Pareek M. Interferon- $\gamma$ release assays: principles and practice. Enferm Infecc Microbiol Clin 2010; 28: 245-252.

3 Andersen P, Munk ME, Pollock JM, et al. Specific immune-based diagnosis of tuberculosis. Lancet 2000; 356 : 1099-1104.

4 Lalvani A, Pareek M. A 100 year update on diagnosis of tuberculosis infection. Br Med Bull 2010; 93: 69-84. Ruhwald M, Ravn P. Biomarkers of latent TB infection. Expert Rev Respir Med 2009; 3: 387-401.

Ruhwald M, Aabye MG, Ravn P. IP-10 release assays in the diagnosis of tuberculosis infection: current status and future directions. Expert Rev Mol Diagn 2012; 12: 175-187.

7 Kasprowicz VO, Halliday JS, Mitchell J, et al. MIGRAs: are they the new IGRAs? Development of monokineamplified IFN- $\gamma$ release assays. Biomark Med 2012; 6: 177-186.

8 Groom JR, Luster AD. CXCR3 ligands: redundant, collaborative and antagonistic functions. Immunol Cell Biol 2011; 89: 207-215.

9 Guzzo C, Che Mat NF, Gee K. Interleukin-27 induces a STAT1/3- and NF-kB-dependent proinflammatory cytokine profile in human monocytes. J Biol Chem 2010; 285: 24404-24411.

10 Rudner XL, Happel KI, Young EA, et al. Interleukin-23 (IL-23)-IL-17 cytokine axis in murine Pneumocystis carinii infection. Infect Immun 2007; 75: 3055-3061.

11 Liu M, Guo S, Hibbert JM, et al. CXCL10/IP-10 in infectious diseases pathogenesis and potential therapeutic implications. Cytokine Growth Factor Rev 2011; 22: 121-130.

12 Aabye MG, Eugen-Olsen J, Werlinrud AM, et al. A simple method to quantitate IP-10 in dried blood and plasma spots. PLoS One 2012; 7: e39228.

13 Mei JV, Alexander JR, Adam BW, et al. Use of filter paper for the collection and analysis of human whole blood specimens. J Nutr 2001; 131: 1631S-1636S.

14 Jepsen M, Krause TG, Moryl D, et al. Sammenligning af QuantiFERON og hudtest ved mulig tuberkuloseinfektion [Comparison of QuantiFERON and tuberculin skin test in possible suspected tuberculosis infection]. Ugeskr Laeger 2009; 171: 2625-2631.

15 Walzl G, Ronacher K, Hanekom W, et al. Immunological biomarkers of tuberculosis. Nat Rev Immunol 2011; 11: 343-354.

16 Ruhwald M, Dominguez J, Latorre I, et al. A multicentre evaluation of the accuracy and performance of IP-10 for the diagnosis of infection with M. tuberculosis. Tuberculosis (Edinb) 2011; 91: 260-267.

17 Ruhwald M, Bodmer T, Maier C, et al. Evaluating the potential of IP-10 and MCP-2 as biomarkers for the diagnosis of tuberculosis. Eur Respir J 2008; 32: 1607-1615.

18 Aabye MG, Ruhwald M, Praygod G, et al. Potential of interferon- $\gamma$-inducible protein 10 in improving tuberculosis diagnosis in HIV-infected patients. Eur Respir J 2010; 36: 1488-1490.

19 Kabeer BS, Sikhamani R, Raja A. Comparison of interferon- $\gamma$ and interferon- $\gamma$-inducible protein-10 secretion in HIV-tuberculosis patients. AIDS 2010; 24: 323-325.

20 Kabeer BS, Sikhamani R, Raja A. Comparison of interferon- $\gamma$-inducible protein-10 and interferon- $\gamma$-based QuantiFERON TB Gold assays with tuberculin skin test in HIV-infected subjects. Diagn Microbiol Infect Dis 2011; 71: 236-243.

21 Vanini V, Petruccioli E, Gioia C, et al. IP-10 is an additional marker for tuberculosis (TB) detection in HIVinfected persons in a low-TB endemic country. J Infect 2012; 65: 49-59.

22 Goletti D, Raja A, Syed Ahamed Kabeer B, et al. Is IP-10 an accurate marker for detecting M. tuberculosis-specific response in HIV-infected persons? PLoS One 2010; 5: e12577.

23 Chen D-Y, Shen G-H, Chen Y-M, et al. Interferon-inducible protein-10 as a marker to detect latent and active tuberculosis in rheumatoid arthritis. Int J Tuberc Lung Dis 2011; 15: 192-200.

24 Lighter J, Rigaud M, Huie M, et al. Chemokine IP-10: an adjunct marker for latent tuberculosis infection in children. Int I Tuberc Lung Dis 2009; 13: 731-736.

25 Ruhwald M, Petersen J, Kofoed K, et al. Improving T-cell assays for the diagnosis of latent TB infection: potential of a diagnostic test based on IP-10. PLoS One 2008; 3: e2858.

26 Yassin MA, Petrucci R, Garie KT, et al. Can interferon- $\gamma$ or interferon- $\gamma$-induced-protein-10 differentiate tuberculosis infection and disease in children of high endemic areas? PLoS One 2011; 6: e23733.

27 Alsleben N, Ruhwald M, Rüssmann $\mathrm{H}$, et al. Interferon- $\gamma$ inducible protein 10 as a biomarker for active tuberculosis and latent tuberculosis infection in children: a case-control study. Scand J Infect Dis 2012; 44: 256-262.

28 Snijdewind IJM, van Kampen JJA, Fraaij PLA, et al. Current and future applications of dried blood spots in viral disease management. Antiviral Res 2012; 93: 309-321.

29 Levy PA. An overview of newborn screening. I Dev Behav Pediatr 2010; 31: 622-631.

30 Skogstrand K, Thorsen P, Nørgaard-Pedersen B, et al. Simultaneous measurement of 25 inflammatory markers and neurotrophins in neonatal dried blood spots by immunoassay with xMAP technology. Clin Chem 2005; 51: $1854-1866$.

31 Eising S, Svensson J, Skogstrand K, et al. Type 1 diabetes risk analysis on dried blood spot samples from population-based newborns: design and feasibility of an unselected case-control study. Paediatr Perinat Epidemiol 2007; 21: 507-517. 

tuberculosis infection using adsorption of stimulated whole blood on filter paper and multiplex analysis. Scand J Clin Lab Invest 2012; 72: 204-211.

33 Skogstrand K. Multiplex assays of inflammatory markers, a description of methods and discussion of precautions our experience through the last ten years. Methods 2012; 56: 204-212.

34 Ruhwald M, Andersen ES, Christensen PB, et al. IP-10 can be measured in dried plasma spots in patients with chronic hepatitis C infection. PLoS One 2012; 7: e45181.

35 Bélard E, Semb S, Ruhwald M, et al. Prednisolone treatment affects the performance of the QuantiFERON gold intube test and the tuberculin skin test in patients with autoimmune disorders screened for latent tuberculosis infection. Inflamm Bowel Dis 2011; 17: 2340-2349.

36 Pai M, Dheda K, Cunningham J, et al. T-cell assays for the diagnosis of latent tuberculosis infection: moving the research agenda forward. Lancet Infect Dis 2007; 7: 428-438.

37 Zhou X-H, Obuchowski NA, McClish DK. Statistical Methods in Diagnostic Medicine. 1st Edn. Hoboken, WileyInterscience, 2002.

38 Menzies D, Pai M, Comstock G. Meta-analysis: new tests for the diagnosis of latent tuberculosis infection: areas of uncertainty and recommendations for research. Ann Intern Med 2007; 146: 340-354.

39 Mori T. Specific detection of tuberculosis infection: an interferon- $\gamma$-based assay using new antigens. Am J Respir Crit Care Med 2004; 170: 59-64.

40 Streeton JA, Desem N, Jones SL. Sensitivity and specificity of a $\gamma$-interferon blood test for tuberculosis infection. Int J Tuberc Lung Dis 1998; 2: 443-450.

41 Syed Ahamed Kabeer B, Raman B, Thomas A, et al. Role of QuantiFERON-TB Gold, interferon $\gamma$ inducible protein-10 and tuberculin skin test in active tuberculosis diagnosis. PLoS One 2010; 5: e9051.

42 Kabeer BSA, Raja A, Raman B, et al. IP-10 response to RD1 antigens might be a useful biomarker for monitoring tuberculosis therapy. BMC Infect Dis 2011; 11: 135. 